\title{
ANÁLISE FARMACOGNÓSTICA DO PÓ E AVALIAÇÃO DO EFEITO DO EXTRATO ETANÓLICO DAS FLORES DA Calendula officinalis L. CULTIVADAS NO BRASIL NO SISTEMA IMUNOLÓGICO
}

\author{
Pharmacognostic analysis of the dust and evaluation of the effect of the ethanolic extract of the \\ flowers of Calendula officinalis I. cultivated in Brazil in the immune system
}

\author{
Parente, I. M. I. ${ }^{1}$; Matos, I. G. ${ }^{2}$; Cunha, L.C. ${ }^{3}$; Leão, A. R. ${ }^{4}$; Paula, J. R. ${ }^{5}$; Júnior, G.V. ${ }^{6}$; \\ Silveira, N. A. \\ ${ }^{1}$ Acadêmica do curso de Mestrado em Biologia do Instituto de Ciências Biológicas - UFG. Brasil. \\ ${ }^{2}$ Técnica do Laboratório de Produtos Naturais do Instituto de Ciências Biológicas - UFG. Brasil. \\ ${ }^{3}$ Prof. Dr. Do Departamento de Toxicologia da Faculdade de Farmácia - UFG. Brasil. \\ ${ }^{4}$ Acadêmica do curso de Mestrado em Biologia do Instituto de Ciências Biológicas - UFG. Brasil. \\ ${ }^{5}$ Prof. Dr. Do Departamento de Farmacognosia da Faculdade de Farmácia - UFG. Brasil. \\ ${ }^{6}$ Acadêmico do curso de Mestrado em Biologia do Instituto de Ciências Biológicas - UFG. Brasil. \\ ${ }^{7}$ Profa.Dra. do Departamento de Fisiologia do Instituto de Ciências Biológicas - UFG. Brasil.
}

Recebido em 26/10/2204 - Aceito em 23/11/2004

\begin{abstract}
RESUMO: Calendula officinalis L. (Asteraceae) é uma herbácea anual originada da área do Mediterrâneo e adaptada ao Brasil. Muitos fatores relacionados ao cultivo podem afetar de diferentes de forma qualidade e quantidade dos princípios ativos produzidos pela planta. Nesse trabalho, foi realizada uma avaliação farmacognóstica das flores da C. officinalis do Brasil (Paraná) como controle de qualidade do material botânico. A planta, conhecida há muito tempo, apresenta atividades antiinflamatórias e cicatrizante, entre outras, e está presente em muitas formulações terapêuticas comercializadas. Nesse trabalho, foi obtido um extrato etanólico das flores da $C$. officinalis (EEC) cultivadas no Brasil e realizou-se uma avaliação do efeito do EEC no sistema imunológico, verificando se o extrato obtido também apresentaria atividade imunomoduladora relatada para frações isoladas da planta cultivada na Europa e Ásia.
\end{abstract}

ABSTRACT: Calendula officinalis L. (Asteraceae) is an annual herbaceous from Mediterranean area and it was adapted in Brazil. Lots of factors related to cultivation, can affect in a significant way the quality and the quantity of the active principles produced by the plants. In this work, pharmacognostic evaluation of the flowers of $C$. officinalis from Brazil (Paraná), was accomplish for the quality control of the botanical material. The plant, well known a long time ago, has anti-inflammatory and healing activities and several other effects. It is present in many commercialized therapeutic formulations. In this work, an ethanolic extract of the flowers of the cultivated $C$. officinalis (EEC) in Brazil was gotten and was become an evaluation of the effect of the EEC in the immunologic system, verifying if the extract also gotten it would present the same immunomodulatory activity for isolated fractions of the plant cultivated in the Europe and Asia.

KEYWORDS: Calendula officinalis, pharmacognostic evaluation, immunomodulatory activity 


\section{INTRODUÇÃO}

A Calendula officinalis L. (C. officinalis) pertence à família Asteracea (Compositae). É uma herbácea anual com cerca de $50 \mathrm{~cm}$ de altura com folhas grossas, verdes, inteiras e pilosas (SILVA et al, 1995). Apresenta flores dispostas em capítulos com 3 a 7cm, liguladas na periferia, com corolas amareladas ou alaranjadas (FARMACOPÉIA BRASILEIRA IV, 2001).

As flores liguladas da $C$. officinalis são usadas em formas terapêuticas e em cosméticos, as quais apresentam principalmente: saponinas, triterpenos, triterpenos alcóois e ésteres de ácidos graxos, carotenóides, flavonóides, cumarinas, óleos essenciais, hidrocarbonetos e ácidos graxos (HAMBURGUER et al, 2003). A descrição detalhada dos componentes químicos da C. officinalis foi feita por ALONSO (1998). Os óleos essenciais apresentam uma grande quantidade de mono e sesquiterpenos oxigenados, compostos principalmente por: carvona, geranilacetona, mentona, isomentona, cariofileno, $\alpha$ e $\beta$-iononas, penduculatina e dihidroactinidiólido. Os carotenóides, compostos relativamente estáveis, solúveis em gordura e insolúveis em água, apresentaram calendulina, caroteno, licopeno, rubixantina, violaxantina, zeína entre outros. As saponinas são calendulosídios A, $\mathrm{D}, \mathrm{D}_{2}, \mathrm{~F}$. Os flavonóides encontrados foram os derivados do quercetol (quercetin-3-o-glicídio) e do isorramnetol. Os álcoois triterpenos pentacíclicos apresentaram principalmente: arnidiol, faradiol, ácido faradiol-3-mirístico, lupeol, taraxasterol e ácido faradiol-3- palmítico. Outros componentes foram encontrados, como: ácido málico, mucilagem, resina, goma (calendulina), substância amarga (calendeno e calendina), tanino, poliacetilenos, esteróis sitoesterois, estigmasterol, isofucosterol, campesterol, metil-(enecolesterol e colesterol), ácido salicílico e inulina (raíz) (ALONSO,1998).

O uso medicinal da planta remonta à época da antiguidade (ALONSO, 1998). Muitas propriedades medicinais vêm sendo atribuídas às flores da planta, dentre elas: colerética, antiinflamatória, analgésica, antitumoral, bactericida, diurética, cicatrizante, sedativa e imunomoduladora (FRANCO,1996; BROWN \& DATTNER, 1998; RAMOS et al, 1998).

A fração polissacarídea da $C$. officinalis, derivada do grupo dos heteroglocanos, apresentou atividade imunomoduladora in viro (ALONSO, 1998). Três polissacarídeos foram isolados através do fracionamento em etanol e filtração em gel. Essa porção polissacarídea da planta apresentou atividade antitumoral (MANOLOV apud VARLJEN et al, 1989). Acredita-se que a planta estimule a granulação e aumente o metabolismo das glicoproteínas e do colágeno no local do ferimento (BROWN \& DATTNER, 1998).

A C. officinalis é uma espécie originária do Mediterrâneo, domesticada e adaptada ao Brasil. No processo de cultivo, alguns fatores podem afetar de forma significativa a qualidade e a quantidade de princípios ativos produzidos pelas plantas, pois rotas metabólicas podem ser ativadas e/ou inativadas, levando à produção de diversos produtos do metabolismo secundário em cada situação (SILVA et al,1995).

Este trabalho realizou uma avaliação farmacognóstica das flores pulverizadas da planta cultivada no Brasil (Paraná), para realizar um controle de qualidade do material botânico. Foi obtido o extrato etanólico das flores da C. officinalis (EEC) e realizou-se uma avaliação do efeito do EEC no sistema imunológico, verificando se o extrato obtido também apresentaria atividade imunomoduladora relatada para frações isoladas da planta cultivada na Europa e Ásia.

\section{MÉTODOS}

\section{Material botânico}

Flores pulverizadas da C.officinalis foram adquiridas da Empresa Clorophila, sediada em Goiânia, Goiás.

\section{Avaliação Farmacognóstica}

\section{Propriedades organolépticas}


Foram observados a coloração e o odor das flores pulverizadas da C. officinalis (FARMACOPÉIA BRASILEIRA IV, 2001).

\section{Descrição microscópica}

A avaliação microscópica das flores pulverizadas da C. officinalis foi realizada com o reagente de Steinmetz (COSTA, 1982) e de Etzold (ETZOLD \& KONTTRATEICHE, 1983).

\section{Cromatografia em camada delgada}

A identificação das flores pulverizadas foi realizada através da Cromatografia em camada delgada. $O$ teor de flavonóides totais foi expresso em hiperosídeo de acordo com técnica da FARMACOPÉIA BRASILEIRA IV (2001).

Teor de cinzas totais

O teor de cinzas totais foi determinado em triplicata por incineração das cinzas a $450^{\circ} \mathrm{C}$ (COSTA, 1982; PREGNOLATTO \& PREGNOLATTO, 1985).

\section{Produção do Extrato Etanólico das flores da C. officinalis (EEC)}

O extrato etanólico das flores da $C$. officinalis foi produzido por maceração á frio e concentrado em evaporador rotativo.

\section{Avaliação do Efeito do EEC no Sistema Imunológico}

\section{Animais}

Camundongos machos albinos-Swiss (20-30g), fornecidos pelo Biotério Central da Universidade Federal de Goiás, foram usados nos experimentos.

\section{Teste Geral de Atividade Farmacológica em camundongos}

Grupos de camundongos $(n=5)$ foram tratados pelas vias oral, intraperitoneal e subcutânea com EEC nas doses de 0,1, 0,3 e 1 $\mathrm{g} / \mathrm{kg}$. O grupo controle foi tratado com água destilada, utilizada na diluição do EEC, em volume proporcional às doses utilizadas. Os efeitos observados foram registrados em ficha padrão de triagem farmacológica (MALONE, 1977).

\section{Gaiolas metabólicas}

Grupos de animais $(n=10)$ foram colocados individualmente em gaiolas metabólicas, tratados diariamente por via oral (gavagem), nas doses de 0,1, 0,3 e 1,0 g/Kg de EEC e com o veículo da diluição do EEC (água destilada), durante um período de quatro semanas. Diariamente foram registrados para cada animal: o volume de água ingerida, o peso da ração consumida, o volume de urina excretada e o peso das fezes produzidas. Em intervalos de sete dias, os animais foram pesados para a determinação da evolução de peso corporal durante o período experimental. Os animais foram sacrificados através de punção cardíaca no $29^{\circ}$ dia. Coletou-se sangue para realização de exames hematológicos que constaram do hemograma completo.

\section{Análise Estatística}

Os resultados foram expressos como médias \pm erro padrão das médias, submetidos à análise de variância (ANOVA). O teste " $t$ " de Student permitiu comparar as médias dos grupos experimentais com o grupo controle. Foram considerados significativos valores de $p<0,05$ (CRESPO, 2001). 


\section{RESULTADOS E DISCUSSÃO}

$\mathrm{Na}$ avaliação farmacognóstica, observou-se que as flores pulverizadas da $C$. officinalis apresentaram uma coloração amarelada e um odor suave e levemente aromático. As lâminas do pó das flores da planta coradas com os reagentes de Steinmetz e de Etzold permitiram identificação de: tricoma multicelular bisseriado do tubo da corola da flor ligulada; epiderme da lígula com cutícula estriada; parênquima da lígula contendo gotas de material lipídica; grãos do pólen. Na cromatografia em camada delgada usou-se uma mistura padrão de rutina (Rf $-0,43)$ de quercetina e ácido cafeico (Rf - 0,88). Na amostra observou-se: mancha correspondente a rutina (Rf $-0,47)$, mancha amarelada correspondente a flavonóides ( $R f-0,29)$, mancha com fluorescência azul $(R f-0,8)$ e mancha amarelada correspondente a agliconas de flavonóides ( $\mathrm{Rf}-0,92)$. $\mathrm{O}$ teor de flavonóides totais das flores pulverizadas foi de $0,77 \%$ e no EEC foi de $1,29 \%$. O teor de cinzas totais foi de $8,47 \%$.

No teste geral de atividades farmacológicas foi observado, uma hora após o tratamento, um óbito na dose de $1 \mathrm{~g} / \mathrm{kg}$ do EEC administrado pela via intraperitoneal (i.p.). Não foram observadas alterações quanto aos parâmetros: sialorréia, diâmetro pupilar, fasciculações, tremor de cauda, dispnéia, alterações nas orelhas (palidez, cianose e hiperemia), diarréia, contorções, agressividade, freqüência urinária, entre outros.

O tratamento com EEC na dose de 1g/Kg diminuiu significativamente o número de linfócitos para 82,6 $\pm 5,2 \%$ em relação ao controle $(80,4 \pm 2,3 \%)$ (Figura 1 ,Tabela 1$)$. O tratamento com EEC na dose de $1 \mathrm{~g} / \mathrm{Kg}$ aumentou significativamente o número de neutrófilos totais para 190,2 $\pm 24,5 \%$ em relação ao controle (16,3 \pm 11,8\%) (Figura 2,Tabela 2).

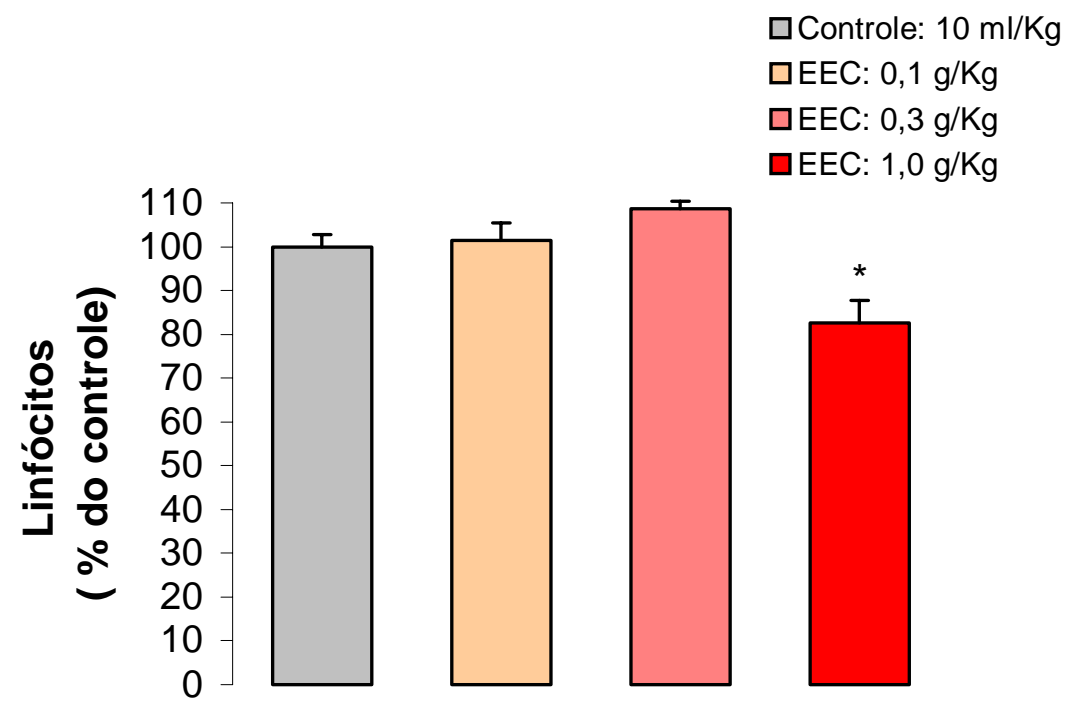

Figura 1. Efeito do tratamento (28 dias) com extrato etanólico das flores da C. officinalis (EEC - 0,1, 0,3 ou 1g/Kg) no número de linfócitos dos ratos. As barras verticais indicam as médias \pm erro padrão das médias de 10 animais por grupo experimental, expressas em porcentagem relativas ao grupo controle.

* Estatisticamente diferente do grupo controle $(p<0,05)$.

Tabela 1. Efeito do tratamento (28 dias) com EEC na taxa de linfócitos (\%) em ratos ( $n=10)$.

\begin{tabular}{|c|c|c|c|}
\hline Tratamento & Dose e via & Linfócitos (\%) & \% diferença \\
\hline Controle & 10mL/kg, v.o. & $80,4 \pm 2,3$ & $100,0 \pm 2,9$ \\
\hline \multirow[t]{3}{*}{ EEC } & 0,1 g/kg, v.o. & $81,5 \pm 3,2$ & $101,4 \pm 4,2$ \\
\hline & 0,3 g/kg, v.o. & $87,4 \pm 1,9$ & $108,7 \pm 1,2$ \\
\hline & 1,0 g/kg, v.o. & $66,4 \pm 4,2^{*}$ & $82,6 \pm 5,2$ \\
\hline
\end{tabular}


Os valores representam a média \pm e.p.m. da taxa de linfócitos (\%) e a porcentagem da diferença e do aumento relativos ao grupo controle $(n=10)$.

${ }^{*} p<0,05$ quando comparado com o veículo (ANOVA, Teste $t$ de Student).

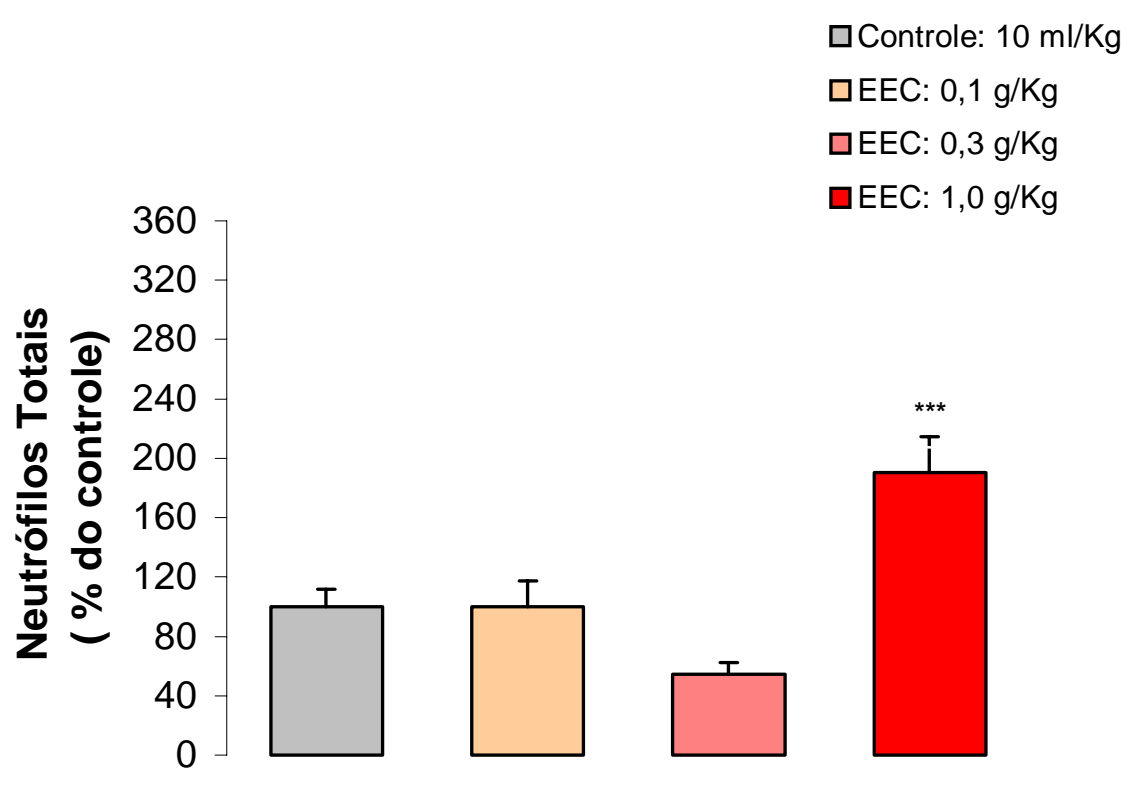

Figura 2. Efeito do tratamento (28 dias) com extrato etanólico das flores da C. officinalis (EEC - 0,1, 0,3 ou 1g/kg) no número de neutrófilos totais dos ratos. As barras verticais indicam as médias \pm erro padrão das médias de 10 animais por grupo experimental, expressas em porcentagem relativas ao grupo controle.

*** Estatisticamente diferente do grupo controle $(p<0,001)$.

Tabela 2. Efeito do tratamento (28 dias) com EEC na taxa de neutrófilos totais (\%) em ratos ( $n=10)$.

\begin{tabular}{|c|c|c|c|}
\hline Tratamento & Dose e via & $\begin{array}{l}\text { Neutrófilos } \\
\text { totais (\%) }\end{array}$ & \% diferença \\
\hline Controle & 10mL/kg, v.o. & $16,3 \pm 11,8$ & $100,0 \pm 11,7$ \\
\hline \multirow[t]{3}{*}{ EEC } & 0,1g/kg, v.o. & $16,3 \pm 2,8$ & $100,0 \pm 17,2$ \\
\hline & 0,3g/kg, v.o. & $8,9 \pm 1,3$ & $54,6 \pm 7,7$ \\
\hline & 1,0g/kg, v.o. & $31,0 \pm 4,0 * * *$ & $190,2 \pm 24,5$ \\
\hline
\end{tabular}

Os valores representam a média \pm e.p.m. da taxa de neutrófilos totais (\%) e a porcentagem da diferença e do aumento relativos ao grupo controle $(n=10)$.

*** $p<0,001$ quando comparado com o veículo ( ANOVA, Teste $t$ de Student).

\section{DISCUSSÃO}

A diagnose do material vegetal foi realizada através de procedimentos específicos descritos para a identificação da $C$. officinalis. Flores pulverizadas da planta apresentaram uma coloração amarelada e um odor suave e levemente aromático. Essas propriedades organolépticas estão de acordo com a descrição na literatura (WHO, 2002). Na descrição microscópica do pó, as duas colorações, de Steinmetz e de Etzol, permitiram a identificação das mesmas estruturas, as quais encontram-se descritas na Farmacopéia Brasileira IV (2001) para essa espécie vegetal. Os resultados obtidos na cromatografia em camada delgada foram compatíveis com a descrição da FARMACOPÉIA BRASILEIRA IV (2001). O teor de flavonóides totais das flores pulverizadas foi de $0,77 \%$ e no EEC foi de $1,29 \%$. Monografias da OMS (WHO, 2002) descrevem que a C. officinalis não deve apresentar menos que $0,4 \%$ de flavonóides totais calculados como hiperosídeo. Assim, os resultados obtidos estão de acordo com o descrito na literatura para esta matéria-prima vegetal. O teor de cinzas totais foi de $8,47 \%$. 
Esse resultado está de acordo com especificações que permitem até 10\% de cinzas totais (FARMACOPÉIA BRASILEIRA IV, 2001; WHO, 2002). Assim, o material botânico testado corresponde às flores pulverizadas da $C$. officinalis.

A partir dos resultados obtidos no teste geral de atividade farmacológica geral optou-se pela utilização da via oral para a administração do EEC, nas doses de 0,1, 0,3 e 1 $\mathrm{g} / \mathrm{Kg}$.

Os hemogramas realizados indicaram que o tratamento com EEC alterou o número de neutrófilos totais e de linfócitos. Os exames dos leucócitos fazem parte do hemograma completo e incluem: determinação quantitativa dos leucócitos, total e diferencial, e avaliação qualitativa de alterações morfológicas celulares. $\mathrm{O}$ tratamento com EEC diminuiu o número de linfócitos, podendo indicar um efeito imunodepressor do extrato. Por outro lado, aumentou o número de neutrófilos totais (granulocitose), indicando um efeito imunoestimulante, confirmando relatos anteriores da literatura (WAGNER et al, 1985; PATRICK et al, 1996; KALVATCHEV et al, 1997; TESKE \& TRENTINI, 1997; ALONSO, 1998; BROWN \& DATTNER, 1998; NEWALLI et al, 2002; P/EREZ-CARRÉO et al, 2002; GUTERRES \& ZIEGLER, 2002). Esse efeito foi atribuído principalmente aos polissacarídeos de alto peso molecular e aos flavonóides. Embora os mecanismos de ação não estejam bem esclarecidos, acredita-se que a planta atue aumentando a fagocitose (VARLJEN et al, 1989).

Os polissacarídeos são polímeros de alto peso molecular, formados a partir da condensação de um grande número de moléculas de aldoses e cetoses, ocorrendo em bactérias, fungos, algas e vegetais superiores. Polissacarídeos de origem vegetal podem apresentar produtos naturais bioativos com atividades antitumoral, imunoestimulante, anticomplemento, antiinflamatória, antiviral, hipoglicemiante e hipocolesterolemiante, como já relatado (Poser et al, 1999). Os flavonóides são os compostos naturais mais disseminados em plantas, seja em estado livre como em glicerídeos. O emprego em terapêutica desses compostos é muito grande e vem sendo feito de forma empírica. Algumas pesquisas sugerem que esses possam apresentar ação anticancerosa considerável, podendo atuar como antiviral e combatendo radicais livres (Robbers et al,1997; Zuanazzi et al, 1999).

Os efeitos aparentemente antagônicos sobre o sistema imunológico que foram observados nos tratamentos dos animais com EEC, estão de acordo com um estudo in vitro realizado por Amirghofran et al (2000), que testou o efeito imunomodulador do extrato etanólico (70\%) de cinco plantas medicinais encontradas no Irã, e entre elas a C. officinalis. Em um dos testes ("MRL Test"), o extrato da planta exerceu efeito estimulante na proliferação de linfócitos, seguido por um efeito inibitório, em altas concentrações. Embora esses dados tenham sido obtidos de um estudo in vitro, foram semelhantes aos obtidos neste estudo na avaliação do efeito imunomodulador in vivo do EEC para os linfócitos.

$\mathrm{Na}$ presente investigação, o efeito imunoestimulante do EEC avaliado através do hemograma dos ratos tratados diariamente não apresentou resultado como esperado para a espécie vegetal e relatado na literatura. Pode-se sugerir que o EEC poderia não apresentar quantidades suficientes dos princípios ativos responsáveis por esse efeito, ou esses compostos poderiam não ocorrer ou ocorre em mínimas proporções nas flores da planta cultivada no Brasil.

\section{CONSIDERAÇÕES FINAIS}

Os dados obtidos não permitem elucidar o efeito que o EEC exerceu sobre o sistema imunológico na metodologia experimental adotada, sendo necessárias outras metodologias para esclarecer a questão, como por exemplo, o teste de esfregaço de granulócitos e a depuração (clerance) do carbono.

\section{REFERÊNCIAS BIBLIOGRÁFICAS}

ALONSO, Jorge R. Tratado de Fitomedicina: Bases clínicas y Farmacológicas. Buenos Aires: Isis Ediciones, 1998, p.327-331.

AMIRGHOFRAN, Zahra; AZADBAKHT, Mohammed; KARIMI, Mohammed H. Evaluation of the immunomodulatory effects of five herbal plants. Journal of Ethnopharmacology. Shiraz, v. 72, p.167-172, 2000. 
BROWN, Donald J.; DATTNER, Alan. Phytoterapeutic Approaches to Common Dermatologic Conditions. Arch of Dermatologic, v.134, p.1401-1404, 1998

COSTA, A. F. Farmacognosia. Lisboa: Fundação Calouste Gulbenkian. Vol. III, 2ª ed., 1982, 1032p.

CRESPO, Antônio Arnot. Estatística Fácil. $17^{\mathrm{a}}$ ed. São Paulo: Editora Saraiva, 2001, 150p.

ETZOLD, H.; KONTRASTREICHE, Elina. Simultane mehrefachfarbung fur pflanzenanatomiche proparate . Mirkroskosmos, 72, p. 213-218, 1983.

FARMACOPÉIA BRASILEIRA IV. São Paulo: Editora Ateneu, 2001, p.134.

FRANCO, Lelington Lobo. As Sensacionais 50 Plantas Medicinais campeãs de poder curativo.Curitiba: Editora Santa Mônica, 1996. 241p.

GUTERRES, Silva; ZIEGLER, Sabine. Matérias-primas vegetais com ação antiinflamatória empregadas em produtos dermo-cosméticos. Infar, v.14, n.1/2, p.79-83, 2002.

HAMBURGUER, M.; ADLER, S.; BAUMANN, D.; FORG, A.; WEINREICH, B. Preparative purification of the major anti-inflamatory triterpenoid ester from Marigold (Calendula officinalis). Fitoterapia, v.74, p.328-338, 2003

KALVATCHEV, Z.; WALDER,R.; GARZARO, D. Anti-HIV activity of extracts from Calendula officinalis flowers. Biomed \& Pharmacother.Venezuela, v.51, p.176-180, 1997.

MALONE, M.H. Pharmacological approaches to natural products, screening and evaluation. New natural pruducts and plants drugs with pharmacological, biological or therapeutical activity. Berlin: Edited by H. Eagner and P.Wolf Springer - Verlag, 1977, p. 24-53.

NEWALL, Carol A.; ANDERSON, Linda A.; PHILLIPSON, J. David. Plantas Medicinais : guia para profissional de saúde. São Paulo: Ed. Premier, 2002, 312 p.

PATRICK, K.F. M.; KUMAR, S.; EDWARDSON, P.A.D.; HUTCHINSON, J.J. Induction of vascularisation by an aqueous extract of the flowers of Calendula officinalis L., the European marigold. Phytomedicine, vol.3, issue 1, 1996, p.11-18.

PÉREZ-CARRÉON, J.I.; CRUZ-JIMÉNEZ, G.; LICEA-VEJA, J.A.; POPOCA, Arce E.; FAZENDA, Fattel S.; VILLATRVIÑO, S. Genotoxic and Anti-genotoxic properties of Calendula officinalis extracts in rat liver cell cultures treated with diethylnitrosamine. Toxicology in vitro, issue 16, p.253-258, 2002.

RAMOS, A.; EDREIRA, A.; VIZOSO, A.; BETANCOURT, J.; LÓPEZ, M.; DÉCALO, M. Genotoxicity of an extract of Calendula officinalis . Journal of Ethnopharmacology. Havana, v. 61, p. 49-55, 1998.

ROBBERS, James E.; SPEEDIE, Marlyn K.; TYLER, Varro E. Farmacognosia e Farmacobiotecnologia. São Paulo: Editora Premier, 1997, 372p.

SILVA, Irene; FRANCO, Selma Lucy; MOLINARI, Sônia Lucy; CONEGERO, Celso Ivan; NETO, Marcílio Hubner de Miranda; CARDOSO, Mara Lane Carvalho; SANT'ANA, Débora de Mello Gonçalves; IWANKO, Neide Salete. Noções sobre organismo humano e utilização de Plantas Medicinais. 4. ed. Cascavel: Assoeste, 1995, 203p.

TESKE, Magrid; TRENTINI, Anny Margaly Maciel. Herbárium: compêndio de fitoterapia. $3^{\mathrm{a}}$ edição. Curitiba: Ingra Editora, 1997, p.66-67.

POSER, Gilsane Lino. Polissacarídeos. In: SIMÕES, Cláudia Maria Oliveira (Org.) et al. . FARMACOGNOSIA, da planta ao medicamento. $4^{\mathrm{a}}$ ed. Florianópolis: Editora da UFSC, 1999, p. 197-220. 
PREgnOlAtTO, W.; PREgnOllatO, N.P. Normas Analíticas do Instituto Adolf Lutz. Vol.I, $3^{\mathrm{a}}$ ed. São Paulo: Impressa Oficial do Estado, 1985, 533p.

VARLJEN, Jadranka; LIPPTAK, Andras; WAGNERT, Hildebert. Structural analysis a rhamnoarabiainogalactatan and arabinogalactans with immuno-stimulating activity from Calendula officinalis. Phytochemistry Riijeka, vol.28, n.9, p.2379-2383, 1989.

ZUANAZZI, José Angelo da Silveira. Flavonóides. In: SIMÕES, Cláudia Maria Oliveira (Org.) et al. . FARMACOGNOSIA, da planta ao medicamento. 4ª ed. Florianópolis: Editora da UFSC, 1999, p. $197-220$.

WAGNER, H.; HIKINO, Hiroshi; FARNSWORTH, Norman R. Economic and Medicinal Plant Research. vol.1. London: Academic Press, 1985, p. 133-143.

WHO Monographs on: Selected medicinal plants. Geneve, vol. 2, 2002, p. 35-44. 